Original Paper http://ajol.info/index.php/ijbcs http://indexmedicus.afro.who.int

\title{
Inventaire et essais phytochimiques sur quelques plantes du Tchad utilisées dans le traitement de la goutte
}

\author{
Séverin MBAÏHOUGADOBE ${ }^{1,2}$, Adolphe Christian NGAKEGNI-LIMBILI ${ }^{1}$, \\ Tsiba GOUOLLALY ${ }^{1}$, Paul NGAÏSSONA ${ }^{1}$, Jean Noel KOANE ${ }^{1}$, \\ Loumpangou Célestine NKOUNKOU ${ }^{1}$, Yaya MAHMOUT ${ }^{2 *}$ et \\ Jean Maurille OUAMBA ${ }^{1}$ \\ ${ }^{1}$ Laboratoire de Chimie du Végétal et de la Vie, Faculté des Sciences et Techniques, Université Marien \\ Ngouabi, BP 69, Brazzaville, Congo. \\ ${ }^{2}$ Laboratoire de Recherche sur les Substances Naturelles, Faculté des Sciences Exactes et Appliquées, \\ Université de N'djaména ; BP 1027 N'djaména, Tchad. \\ "Auteur correspondant ; E-mail: yayamahmout@yahoo.fr; Tél. : (+235)66292210
}

\section{RESUME}

La phytothérapie connaît actuellement un regain d'intérêt auprès du public. L'objectif principal de cette étude est d'inventorier les plantes du Tchad utilisées dans le traitement de la goutte. A cet effet, dix-neuf (19) praticiens de la médecine traditionnelle et deux malades ont été interrogés sur la base des fiches d'enquêtes élaborées en vue de recueillir les informations sur les plantes utilisées, les modes de préparation et d'administration des produits. Il ressort de cette enquête que vingt-deux (22) espèces réparties en dix-neuf (19) familles ont été recensées. Par ailleurs, des essais phytochimiques réalisés sur cinq plantes choisies mettant en œuvre les réactions de coloration, ont permis d'identifier sept (7) familles de composés parmi lesquelles cinq (5) d'entre elles (alcaloïdes, acides phénols libres, flavonoïdes, saponosides et triterpènes/stéroïdes) ont été confirmées par chromatographie sur couche mince. La présence de certains groupes de composés a été discutée. Elle confirme ainsi l'utilisation de ces plantes dans le traitement de la goutte.

(c) 2017 International Formulae Group. All rights reserved.

Mots clés : Plantes médicinales, goutte, phytochimie, Tchad.

\section{Inventory and phytochemical screening of some plants used in Chad for the treatment of gout}

\begin{abstract}
The practice of herbal medicine is a worldwide custom that preoccupied mankind in the evolution of most countries. The main objective of this study was to carry out an ethnobotanical survey of plants used in the treatment of gout in Chad. Thus, investigations performed with nineteen (19) traditional healers and two (2) patients allowed to identify twenty-two (22) plants distributed in nineteen (19) different families. Moreover, the phytochemical screening recorded the presence of pharmacologically important substances in five selected species, such as alkaloids, free phenolic acids, flavonoids, saponins, triterpenes derivatives / steroids.
\end{abstract}


Compounds were revealed by thin layer chromatography. The presence of certain groups of compounds in plants was discussed in relation with their use in the treatment of gout.

(C) 2017 International Formulae Group. All rights reserved.

Keywords: Medicinal plants, gout, phytochemistry, Chad.

\section{INTRODUCTION}

La goutte est une maladie métabolique caractérisée par la survenue d'arthrites aiguës récidivantes des articulations périphériques, résultant d'un dépôt intra et péri-articulaire ou tendineux des cristaux d'urate de sodium qui proviennent des liquides organiques saturés en acide urique. Appelée autrefois «maladie des rois ou maladie des riches », la goutte est de nos jours, le plus fréquent des rhumatismes inflammatoires chez l'homme adulte. Ainsi, aux États-Unis, la prévalence dans la population générale est passée de $0,5 \%$ en 1969 à 3\% en 1996. En Angleterre, elle était estimée en 1999 à 1,4\% et atteignait 7\% chez les hommes de plus de 65 ans (Mikuls et al., 2005). On constate de nos jours que la fréquence de la goutte augmente dans certaines parties du monde (Japon, Nouvelle Zélande) (Bardin, 2006) ; au Tchad, en 2012, les services de consultation de référence hospitalière ont notifié 76 nouveaux cas soit une augmentation de 38,18\% par rapport à 2011 (Ministère de la Santé Publique-Tchad, 2012).

Deux modes de traitement de la goutte sont généralement appliqués : le traitement de fond qui ralentit ou réduit l'infiltration uratique de l'organisme et le traitement des accès (Lioté et Bardin, 2007). Dans les deux cas de figures, ces traitements s'accompagnent toujours des effets secondaires néfastes pour le patient (Hammer, et al., 2001 ; Riegersperger et al., 2011). Selon les premières recommandations de l'European League Aganist Rheumatism, (EULAR) le traitement optimal de la goutte doit associer des mesures pharmacologiques et non pharmacologiques. Il est prouvé qu'une telle combinaison est plus efficace que la monothérapie (Zhang et al., 2006).
L'utilisation des plantes médicinales dans le traitement de l'hyperuricémie et de la goutte est basée sur l'expérience de la médecine traditionnelle (Umamaheswari et al., 2007). Ces plantes peuvent être utilisées en application externe, pour soulager les symptômes, et à l'interne pour rétablir l'équilibre dans le corps.

Dans ce présent travail, nous nous proposons d'inventorier les plantes utilisées dans le traitement de la goutte au Tchad et de caractériser les grands groupes chimiques présents dans les espèces les plus citées.

\section{MATERIEL ET METHODES \\ Enquête ethnobotanique}

L'enquête ethnobotanique a été réalisée d'une part, auprès des praticiens de la médecine traditionnelle exerçant dans la ville de Moundou et ses environs, d'autre part, auprès des malades: dix-neuf (19) tradipraticiens et deux (2) malades ont été concernés. Pour s'enquérir de leurs méthodes de traitement des maladies, un questionnaire a été élaboré auquel les réponses ont été fournies. L'identification des espèces a été faite sur la base de comparaison des échantillons avec ceux déposés à l'herbier de l'Institut de Recherche en Élevage pour le Développement (N'Djaména) et confirmée par Docteur Jean-Marie MOUTSAMBOTE, botaniste tropical à l'Ecole Normale Supérieure d'Agronomie et de Foresterie de l’Université Marien Ngouabi.

\section{Matériel végétal}

Les échantillons des plantes ont été récoltées au sud de la ville de Moundou dans le Logone Occidental entre les villages Mankou et Faya (deux villages du canton Boro dans la région du Logone oriental) en 
décembre 2012. Ils ont été découpés en petits morceaux et séchés à la température ambiante à l'abri du soleil. Ensuite, ils ont été broyés, puis pulvérisés à l'aide d'un broyeur mécanique. Les poudres obtenues ont été mises dans des bocaux en verre étiquetés et bien fermés et stockées à l'abri de la lumière pour une utilisation ultérieure. Plusieurs critères ont été pris en compte afin de sélectionner les plantes pour étude, notamment: l'identification univoque de la plante, la facilité d'approvisionnement, l'efficacité reconnue par l'usage traditionnel, l'absence d'étude sur leurs effets antigoutteux et les apports de la littérature. Sur la base des critères ci-dessus énumérés, cinq (5) plantes ont été sélectionnées et ont fait l'objet d'étude. Il s'agit :

- d'écorces de tronc de Combretum Nigricans Lepr. ex Guill, de Crossopteryx febrifuga (AFZ. Ex G. Don) Benth et d'Entada africana (Guill et Perr) ;

- des écorces de racine d'Hexalobus monopetalus (A. Rich.) Engl. \& Diels et de Securidaca longepedunculata Fres.

\section{Tests phytochimiques}

Deux méthodes qualitatives de mise en évidence de familles chimiques ont été employées :

\section{Criblage chimique}

L'analyse phytochimique sommaire a été réalisée sur les différents extraits des écorces des cinq plantes. Les différents groupes de composés présents dans les extraits ont été mis en évidence sur la base des tests de coloration caractéristiques (Bouquet, 1972 ; Bouquet et Debray, 1974).

\section{Chromatographie sur couche mince}

En vue de confirmer les résultats obtenus à partir des tests de coloration, les différents extraits ont été soumis à l'analyse par chromatographie sur couche mince (CCM), réalisée sur les plaques de silice 60 $\mathrm{F}_{254}$ (MercK). Différents systèmes d'élution ont été utilisés en fonction des types de familles recherchées.
Après développement, les plaques ont été examinées à la lumière du jour ou sous la lampe UV à $254 \mathrm{~nm}$ et $365 \mathrm{~nm}$. Les spots ont été ensuite révélés par les réactifs classiques de détection préparés selon les méthodes standards (Wagner et Bladt, 1996).

\section{RESULTATS}

Le Tableau 1 fournit la liste des plantes utilisées contre la goutte ainsi que le mode de préparation des remèdes. Vingt-deux (22) plantes réparties en dix-neuf (19) familles et correspondant à vingt-trois (23) recettes ont été répertoriées. Les recettes sont généralement composées pour les unes d'une seule espèce, pour d'autres en assocation d'autres espèces.

Les résultats des tests phytochimiques réalisés sur les différents organes sont consignés dans le Tableau 2. Sept (7) familles chimiques sont mises en évidence dans les échantillons étudiés. Ce sont : les alcaloïdes, les composés réducteurs, les flavonoïdes, les quinones, les saponosides, les tanins et les triterpènes/stéroïdes.

L'examen de ce tableau montre que les composés majoritaires sont les flavonoïdes, les alcaloïdes, les terpènes/stérols et saponosides. Les composés réducteurs et les quinones sont beaucoup plus mis en évidence dans la plante C. febrifuga.

Parallèlement aux tests de coloration, des CCM ont été réalisées visant les groupes chimiques suivants: alcaloïdes, acides phénols libres, flavonoïdes, saponosides et triterpènes/stéroïdes. Les images des chromatogrammes des différentes familles recherchées, les systèmes d'éluant ainsi que les révélateurs utilisés pour chaque famille chimique sont regroupés (Figure 1). Les familles chimiques recherchées sont mises en évidence dans les images des chromatogrammes par leurs fluorescences caractéristiques. 


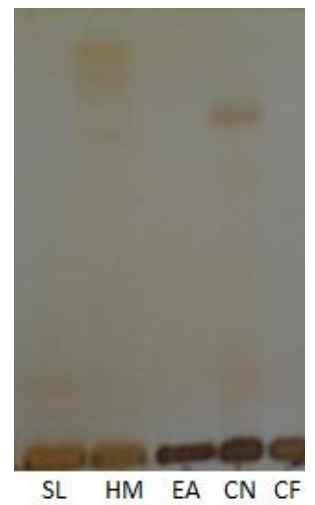

a

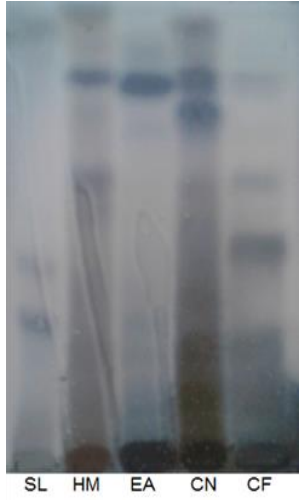

b

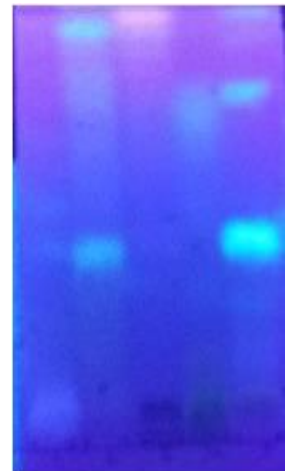

SL HM EA CN CF $\mathrm{C}$

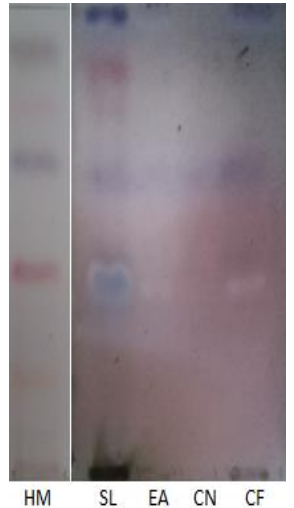

$\mathrm{d}$

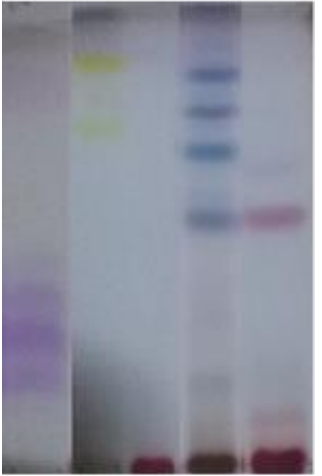

SL HM EA CN CF

$\mathrm{e}$

Figure 1 : chromatogrammes de quelques familles mises en évidence : (a) des alcaloïdes-solvants de migration : Acétate d'éthyle/méthanol/ammoniaque (9/1/1 v/v/v) - extrait Méthanol-révélateur vapeur d'iode; (b) des acides phénoliques libres-solvants de migration : Acétate d'éthyle/Acide formique/eau $(8 / 1 / 1 \mathrm{v} / \mathrm{v} / \mathrm{v})$ - extrait Méthanol-révélateur: Folin ciocalteu suivi de fumigation à l'ammoniaque; (c) des flavonoïdes -solvants de migration : Acétate d'éthyle/Acide formique/eau (8/1/1 v/v/v)- extrait Méthanol-révélateur : NEU (NP/PEG ; (d) des terpènes/stérols -solvants de migration : Toluène/acétate d'éthyle $(8 / 2 \mathrm{v} / \mathrm{v})$ - extrait éther de pétrole-révélateur: anisaldéhyde suivi de chauffage à $110{ }^{\circ} \mathrm{C}$ et (e) des saponosides -solvants de migration : chloroforme/méthanol/eau $(7 / 3 / 0,4)$ - extrait Méthanol-révélateur : anisaldéhyde suivi de chauffage à $110^{\circ} \mathrm{C} . \mathrm{CF}: C$. febrifuga ; $\mathrm{CN}:$ C. nigricans ; EA : E. africana ; HM : H. monopetalus et SL : S. longepedunculata.

Tableau 1 : résultats du criblage phytochimique des extraits.

\begin{tabular}{|c|c|c|c|c|c|c|c|c|c|c|c|c|}
\hline \multirow{2}{*}{\multicolumn{2}{|c|}{ Matériel végétal }} & \multicolumn{11}{|c|}{ Groupes chimiques } \\
\hline & & \multicolumn{2}{|c|}{ Terpènes/stérols } & \multirow[b]{2}{*}{ 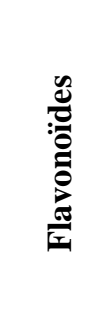 } & \multicolumn{2}{|c|}{ Tanins } & \multirow{2}{*}{ 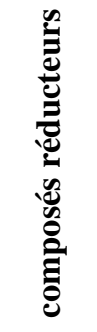 } & \multicolumn{3}{|c|}{ Alcaloïdes } & \multirow[b]{2}{*}{ 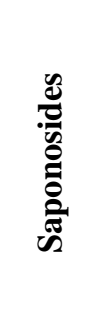 } & \multirow[b]{2}{*}{ 节 } \\
\hline$\frac{\mathscr{E}}{\stackrel{\Xi}{E}}$ & 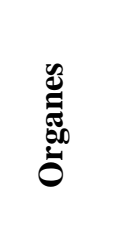 & 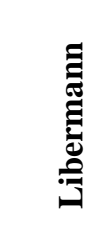 & 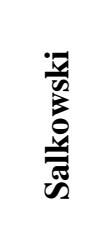 & & 蔦 & 芑 & & $\sum_{\Sigma}^{\bar{\Xi}}$ & 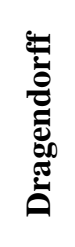 & 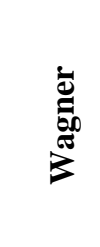 & & \\
\hline $\mathrm{CF}$ & tronc & +++ & + & +++ & + & - & ++++ & - & - & + & +++ & +++ \\
\hline $\mathrm{CN}$ & tronc & +++ & + & ++++ & ++++ & ++ & ++ & + & ++ & ++ & +++ & - \\
\hline E.A & tronc & ++ & ++ & +++ & - & ++++ & - & +++ & + & + & ++ & - \\
\hline $\mathrm{HM}$ & racine & ++ & ++++ & ++++ & +++ & ++++ & - & ++ & + & ++ & + & - \\
\hline SL & racine & +++ & ++ & - & - & - & - & ++++ & ++ & ++++ & ++++ & - \\
\hline
\end{tabular}

Précipité ou coloration : très abondant $:++++$; abondant $:+++;$ moyen $:++$, louche $:+;$ pas de réaction :-. CF : $C$. febrifuga $; \mathrm{CN}:$ C. nigricans $; \mathrm{EA}:$ E. africana $; \mathrm{HM}:$ H. monopetalus et SL : S. longepedunculata. 
Tableau 2 : Liste des plantes du Tchad utilisées dans le traitement de la goutte.

\begin{tabular}{ccc}
\hline $\mathrm{N}^{\circ} \quad$ Famille & Nom de l'espèce & $\begin{array}{c}\text { Mode de préparation et voie d'administration du } \\
\text { remède }\end{array}$ \\
\hline
\end{tabular}

1 Annonacées Hexalobus monopetalus (A.

Rich.) Engl. \& Diels

\begin{tabular}{cccc}
\hline 2 & Apocynacées & $\begin{array}{c}\text { Calotropis procera } \\
\text { (Ait.)Ait.F }\end{array}$ & $\begin{array}{c}\text { La poudre est mélangée avec l'huile de karité. On } \\
\text { masse la partie touchée après chaque bain jusqu'à la } \\
\text { guérison. }\end{array}$ \\
\hline 3 & Burseracées & $\begin{array}{c}\text { Commiphora pedunculata } \\
\text { (Kotschy \& Peyr.) Engl. }\end{array}$ & Faire une décoction et boire deux fois par jour \\
\hline 4 & Caesalpiniacées & Cassia Sieberiana DC. & $\begin{array}{c}\text { Décoction (à partir de 2 verres d'eau, concentré à un } \\
\text { verre). Boire 1 cuillerée à soupe deux fois par jours. }\end{array}$ \\
\hline 5 & Capparacées & $\begin{array}{c}\text { Boscia senegalensis (Pers.) } \\
\text { Lam. Ex Poir. }\end{array}$ \\
\hline
\end{tabular}

6 Caricacées

Carica Papaya

Infusion de feuilles. Boire 2 fois par jour.

\begin{tabular}{|c|c|c|c|}
\hline 7 & Césalniniacées & $\begin{array}{l}\text { Detarium microcarpun (Guill } \\
\text { et Perr.) }\end{array}$ & $\begin{array}{c}\text { Décoction (préparation d'une patte), par cataplasme sur } \\
\text { la partie atteinte. }\end{array}$ \\
\hline 8 & & Senna alata (L.) Roxb. & Décoction, boire un verre par jour. \\
\hline 9 & Combrétacées & $\begin{array}{l}\text { Guiera senegalensis J.F. } \\
\text { Gmel. }\end{array}$ & $\begin{array}{l}\text { Décoction (faire bouillir } 1,5 \text { litre et obtenir } 4 \text { verres) } \\
\text { boire à jeun à partir de } 5 \mathrm{~h}, 2 \text { verres et une heure plus } \\
\text { tard, } 2 \text { verres continuer pendant } 3 \text { jours. }\end{array}$ \\
\hline 10 & & $\begin{array}{l}\text { Combretum nigricans Lepr. } \\
\text { ex Guill. Et Perr. }\end{array}$ & $\begin{array}{c}\text { Décoction d'une cuillerée à soupe de poudre }+ \text { un } \\
\text { demi-verre d'eau et } 3 \text { carreaux de sucre. Boire comme } \\
\text { le thé. }\end{array}$ \\
\hline 11 & Lamiacées & Hoslundia opposita Vahl & $\begin{array}{l}\text { Décoction avec la poudre de Grewia venusta Fresen } \\
\text { (prendre un verre du mélange des deux poudres et deux } \\
\text { litres d'eau qui seront réduits à un litre et demi). Boire } \\
\text { toute la journée et pendant une semaine. On peut aussi } \\
\text { utiliser en cataplasme (mélanger les deux poudres avec } \\
\text { un peu d'eau et bander la partie atteinte). }\end{array}$ \\
\hline 12 & Malavacées & Sterculia setigera Del & $\begin{array}{l}\text { La poudre de la gomme est mélangée avec l'huile de } \\
\text { karité. On masse la partie touchée après chaque bain } \\
\text { jusqu'à la guérison. }\end{array}$ \\
\hline 13 & Mimosacées & $\begin{array}{l}\text { Entada Africana Guill. Et } \\
\text { perr }\end{array}$ & $\begin{array}{c}\text { Décoction dans une jarre, lavé le malade avec un bout } \\
\text { de tissus à minuit. Décoction et boire } 4 \text { à } 5 \text { fois par } \\
\text { jours. }\end{array}$ \\
\hline
\end{tabular}


Décoction avec l'ail. boire un demi-verre matin et soir pendant 3 jours.

14 moracées Ficus gnaphalocarpa (Miq.)

Steud. Er A . Rich.
Piler l'écorce de la racine et du tronc avec l'ail puis masser les parties douloureuses matin et soir. Durée de traitement 3 jours.

15 Moringacées Moringa oleifera (Lam.)

Décoction (prendre un litre d'eau et une cuillerée de la poudre de la graine, les réduire à un demi litre), boire un verre matin, midi et soir

Infusion et boire. Mettre une cuillerée à soupe la poudre des feuilles séchées dans un repas. Faire 3 fois par jours.

\begin{tabular}{|c|c|c|c|}
\hline 16 & Myrtacées & $\begin{array}{c}\text { Syzygium guineense (Willd.) } \\
\text { DC. }\end{array}$ & Décoction, boire et se laver $4 a ̀$ 5 fois par jour. \\
\hline 17 & Phyllanthacées & $\begin{array}{l}\text { Phyllanthus amarus } \\
\text { Schumach. \&Thonn. }\end{array}$ & $\begin{array}{c}\text { Décoction (faire bouillir } 3 \text { verres pour n'en obtenir } \\
\text { qu'un). Boire un verre chaque deux jours pendant un } \\
\text { mois }\end{array}$ \\
\hline
\end{tabular}

18 Polygalacées

Securidaca longepedunculata Fres

Macération de 3 à 4 cuillerées à soupe de la poudre des racines dans un litre. Boire un demi-vert matin et soir jusqu'à la guérison.

Décoction (prendre 3 cuillerées à soupe de la poudre

Crossopteryx febrifuga pour 3 verres ordinaires d'eau et faire bouillir comme le thé). Ajouter un peu de sucre et boire 2 verres par jour pendant 10 à 15 jours.

Rubiacées

20

Mitragyna inermis (Willd.)
O. Ktze

Mitragyna inermis (Willd.)
O. Ktze

O. Ktze

Infusion et boire un verre par jour et ce pendant 3 jours.

Faire une décoction d'une cuillerée à soupe de poudre + un demi-verre d'eau et 3 carreaux de sucre. Boire comme le thé.

Décoction avec la poudre de Hoslundia opposita Vahl (prendre un verre du mélange des deux poudres et deux litre d'eau qui seront réduits à un litre et demi). Boire toute la journée et pendant une semaine. On peut aussi utiliser en cataplasme (mélanger les deux poudres avec un peu d'eau et bander la partie atteinte).

21 Tiliacées Grewia venusta Fresen.

Ampelocissus pentaphylla

22 Vitacées
Gilg \& M.Brandt ou

Ampelocissus multistriata (Baker) Planch.
Une fois les racines réduites en poudre on prépare une bouillie et on applique en cataplasme (masser la partie atteinte) 2 fois par jour. 


\section{DISCUSION}

L'étude ethnobotanique réalisée a permis de comprendre que certaines plantes recensées au Tchad sont signalées pour avoir été utilisées dans le traitement de la même pathologie dans d'autres pays d'Afrique. Il s'agit notamment de Grewia venusta Fresen, de Commiphora pedunculata (Bonnet et al., 2008) et de Moringa oleifera, dont les activité antalgique, anti-inflammatoire ont été aussi été étudiées (Fuglie, 2001; Ndiaye et al., 2016). La grande majorité des plantes citées sont utilisées soit pour atténuer la douleur, les inflammations, les rhumatismes, soit pour la cicatrisation des plaies, le traitement des œdèmes et d'autres maux (Arbonnier, 2000 ; Bonnet et al., 2008). Plusieurs auteurs ont étudié les propriétés pharmacologiques de ces plantes. Ainsi, les activités analgésiques et anti-inflammatoire de Entada africana (Ifeoma et al., 2013), de Crossopteryx febrifuga (Salawu al., 2008) et de Securidaca longepedunculata Fres ont été mises en évidence (Okoli et al., 2006 ; Muanda, 2010).

L'examen des images des chromatogrammes a révélé que les spots caractéristiques des extraits de C. Nigricans Lepr. ex Guill, montrent la présence des composés majoritaires qui sont les alcaloïdes, les flavonoïdes, les saponosides, les tanins et les terpènes. Les taches apparaissant bleues, bleu-noires sont attribuables aux terpènes et aux saponosides sur la Figure 1 (d) et (e) (Bengad, 2009; Denou, 2016). Après révélation à la vapeur d'iode, une fluorescence brune a été observée. Ce qui pourrait justifier la présence des alcaloïdes, laquelle a été mise en évidence par les réactions de coloration. Les acides phénoliques libres ont aussi été détectés : après pulvérisation au réactif de Folin Ciocalteu, des taches bleu-noires sont observées suite à l'exposition de la plaque à la vapeur d'ammoniac.

Les travaux antérieurs réalisés sur $C$. Nigricans Lepr. ex Guill. ont permis d'isoler les triterpènes pentacycliques (triterpénoïdes oléanane : arjungenin, arjun glucoside). Les diterpénoïdes et triterpénoïdes glycosidiques sont connus pour être les principaux constituants du genre Combretum (Simon et al., 2003 ; Amadou et al., 2013).

Les tests préliminaires effectués sur les extraits de C. febrifuga (AFZ. Ex G. Don) Benth montrent une forte présence d'alcaloïdes, de saponosides, de composés réducteurs et de quinones. Les images des plaques chromatographiques se rapportant aux flavonoïdes montrent des taches bleues. Deux taches sont aussi observées sur la plaque qui se rapportant aux acides phénoliques libres. La CCM révèle en outre la présence des acides phénoliques libres, des flavonoïdes et des saponosides. De cette plante, les études antérieures ont mis en évidence les tanins, les terpènes/stéroïdes. Plusieurs composés ont été isolés de cette plantes: un glucoside, la $\beta$ quinovine identique à celle extraite de Mussaenda parviflora (Rubiacées) par Takéda et al. (2002), deux saponines bisdesmosidiques et des traces d'un alcaloïde, la crossopterine de l'écorce. Les saponines isolées de $C$. febrifuga Benth sont légèrement toxiques et possèdent des propriétés antiinflammatoires, analgésiques, antiédémiques, mucolytiques et antiœedémateuses (Boullard, 2001).

Les réactions de caractérisation réalisées sur les extraits d'E. Africana (Guill et Perr) ont permis de déceler la présence des substances suivantes: saponines, tanins, triterpènes, alcaloïdes et flavonoïdes. La CCM a permis de révéler la présence des acides phénoliques libres et des flavonoïdes. Les travaux antérieurs ont mis en évidence la roténone (diterpène oxygéné), une sapogénine triterpénique (acide entagénique) et des polysaccharides (Diallo et al., 2001).

Les extraits d'H. monopetalus (A. Rich.) Engl. \& Diels contiennent d'après la réaction de coloration, des terpènes, des 
flavonoïdes, des tanins et très peu de saponosides. Ces résultats ont été confirmés par CCM. En effet, la CCM des saponosides montre deux fluorescences jaunes après révélation à l'anisaldéhyde; ce qui pourrait montrer la présence dans la plante des saponines de type stéroïdien. La présence des alcaloïdes a été montrée après exposition des plaques à la vapeur d'iode au terme de laquelle plusieurs taches sont observées près $\mathrm{du}$ front de solvant. Plusieurs alcaloïdes indoliques ont été isolés de cette plante (Hamisi et al., 2014 ; Afua et al., 2016).

Les extraits de $S$. longepedunculata Fres renferment des alcaloïdes, des saponosides, des composés réducteurs et des quinones d'après les réactions de coloration. Par CCM, on note la présence des terpènes/stérols et des saponosides après pulvérisation à l'anisaldéhyde et chauffage à $110{ }^{\circ} \mathrm{C}$. La pulvérisation des plaques avec le réactif de Folin Ciocalteu révèle deux taches bleu-noires avant exposition à la vapeur d'ammoniac ( $R f: 0,82$ et 0,6). Il s'agit probablement des acides phénoliques $p$ - et $O$ hydroxybenzène Wagner et Bladt (Bengad, 2009). Divers travaux réalisés sur cette espèce ont mis en évidence des glucides, des sucres réducteurs, des glucosides, des flavonoïdes, des terpènes, des stérols, des saponines, des acides organiques, des résines, les silicates et leurs dérivés (Yang et al., 2001 ; Okoli et al., 2006). Comme la plupart des organes des plantes de la famille des polygalacées, les écorces de $S$. longepedunculata Fres contiennent une proportion importante de salicylate de méthyle (Jayasekara et al., 2012). Nebie et al. (2004) ont isolé le silicate de méthyle. Deux saponines triterpénoïdes nommés Securidacaside A et B ont été isolées de l'extrait méthanolique de la racine (Philip et al., 2009). Des xanthones purs ont également été isolés (Meli et al., 2006).

Cette étude phytochimique montre que les extraits des plantes contiennent majoritairement des flavonoïdes, des terpènes et des saponosides, ce qui pourrait justifier leur utilisation dans le traitement de la goutte. En effet, les polyphénols et surtout les flavonoïdes extraits des végétaux ont de puissantes propriétés antioxydantes capables d'inhiber la formation de radicaux libres, de chélater les ions métalliques et de s'opposer à l'oxydation des macromolécules dont la xanthine oxydase (XO) et l'hypoxanthine (HXO) (Belal et al., 2006 ; Mo et al., 2007 ; Lespade et al., 2010).Toutes les plantes étudiées contiennent des saponosides, vaste groupe d'hétérosides reconnu pour leur propriété anti-inflammatoire, anti-œdémateuse et antalgique. Par ailleurs, les triterpènes mis en évidence par le criblage phytochimique dans les différentes espèces de plantes possèdent ces mêmes effets (Bruneton, 2009).

\section{Conclusion}

L'enquête ethnobotanique réalisée sur les plantes dans le traitement de la goutte au Tchad a permis d'inventorier 22 espèces appartenant à 19 familles. L'étude phytochimique préliminaire a permis de mettre en évidence des familles chimiques dans les différents extraits des plantes. Il s'agit notamment d'alcaloïdes, de flavonoïdes, de terpènes/stéroïdes, de saponosides. Ces différentes familles de composés manifestent des propriétés anti-inflammatoires, analgésiques, antioxydantes, antipyrétiques, diurétiques; ce qui peut justifier l'utilisation de ces plantes dans le traitement de la goutte.

\section{CONTRIBUTIONS DES AUTEURS}

SM a été l'investigateur principal. TG, $\mathrm{ACN}-\mathrm{L}, \mathrm{PN}$ et Koane JNK ont participé à la préparation des extraits et aux criblages phytochimiques. Le traitement de l'enquête ethnobotanique et l'établissement du listing des plantes ont été réalisés avec l'aide de SM, LCN. Les travaux ont été dirigés par YM et JMO. 


\section{CONFLIT D'INTERETS}

Nous déclarons qu'il n'y a aucun conflit d'intérêts lié à ce travail.

\section{REFERENCES}

Afua EM, Mensah MLK, Abraham YM. 2016. Prenylated indole alkaloids from the stem bark of Hexalobus monopetalus. Phytochemistry Letters 16: 108

114. http://dx.doi.org/10.1016.j.phytol.2016.0 3.012

Amadou D, Saotoing P, Tsala DE, Habtemariam S. 2013. Phytochemical Constituents of Combretum Loefl. (Combretaceae). Pharmaceutical Crops, 4: 38-59. DOI : http://dx.doi.org/10.2174/221029060160 6010013

Arbonnier M. 2000. Arbres, Arbustes et Lianes des Zones Sèches d'Afrique de l'Ouest. CIRAD, MNHN, UICN ; 541.

Bardin T. 2006. Epidémiologie de la goutte. Rev Rhum, 74: 147-149. http://dx.doi.org/10.1016.j//rhum/2006.1 2.003

Belal O, Nornisah M, Rashidah AR, Habibah AW. 2006. Natural flavonoïds for treatement of Hyperuricemia, Molecular Docking studies. IFMBE proceeding, 1, Vol. 14, wold Congress on Medical Physic and Biomedical Engineering, Part 3, 178-182.

Bengad A. 2009. Caractérisation phytochimique et activité antioxydante de quelques cultivars de Phoenix dactylifera $\mathrm{L}$. Mémoire de Magistère en biologie, Faculté des Sciences, Université d'Oran Es-Sénia. Algérie.93p

Bonnet P, Pierre G, Arbonnier M. 2008. Ligneux du Sahel V.1.0. Edition Quae. CIRARD.

Boullard B. 2001. Plantes Médicinales du Monde-Croyance et Réalités. ESTEM : Paris ; 636.

Bouquet A. 1972. Plantes médicinales du Congo Brazzaville. Mémoire
O.R.S.T.O.M., $\mathrm{N}^{\circ} 13$, brazzaville Congo. $116 \mathrm{p}$.

Bouquet A, Debray M. 1974. Plantes Médicinales de la Côte d'Ivoire, O. R. S. T. O. M.: Paris, France ; 232 p.

Bruneton J. 2009. Pharmacognosie, Phytochimie, Plantes Médicinales $\left(4^{\text {̀̀me }}\right.$ edn revue et augmentée). Editions Médicales Internationales, Editions Tec \& Doc Lavoisier : Paris ; 1269.

Denou A, Koudouvo K, Haidara M, Togola A, Sanogo R, Essien K, Aklikokou K A., Diallo D, Gbeassor M. 2016. Activité analgésique de quatre plantes utilisées dans la prise en charge traditionnelle du paludisme au Mali et au Togo. Int. J. Biol. Chem. Sci., 10(3): 1342-1349. DOI: http://dx.doi.org/10.4314/ijbcs.v10i3.34

Diallo D, Paulsen BS, Smestad L, Torun HA, Michaelsen TE. 2001. Polysaccharides from the roots of Entada africana Guill. Et Perr., Mimosaceae, with complement fixing activity. Journal of

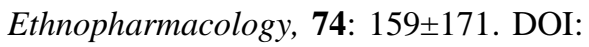
http://dx.doi.org/10.1016/S0378.8741/(0 0)00361-5

Fuglie L. 1999. The Miracle Tree: Moringa oleifera: Natural Nutrition for the Tropics. Church World Service: Dakar; $68 \mathrm{p}$; revised in 2001 and published as the miracle tree: The multiple attributes of Moringa. Church World Service; 172 $\mathrm{p}$.

Ifeoma C Eznyi, Lalasoanirina R, Salawu AO, Emeje M. 2013. Analgesic, antiinflammatory, and heme biomineralization inhibitory properties of Entada africana ethanol leaf extract with antiplasmodial activity against Plasmodium falciparum. Journal of Basic and Clinical Physiology and Pharmacology, 25: 217-223. DOI: https://doi-org.gaelnomade.ujfgrenoble.fr/10.1515/jbcpp-2013-0066

Jayasekara TK, Stevenson P C, Belmain SR, Farman DI, Hall RD. 2002. Identification of methyl salicylate as the principal volatile component in the methanol extract of root bark of Securidaca 
longepedunculata Fers. J. Mass Spectrom; 37: 577-580. http://dx.doi.org/10.1002/jms.314

Hamisi M Malebo, Jonker SA, Waibel R, Mayunga HH. Nkunya. 2014. Diprenylated Indole Alkaloids from Fruits of Hexalobus monopetalus. Nat. Prod. Bioprospect; 4: 101-105. http://dx.doi.org/10.1007/s13659-0140010-x

Hammer B, Link A, Wagner A, Böhm M. 2001. Hypersensitivity syndrome during therapy with allopurinol in asymptomatic hyperuricemia with a fatal outcome. Deutsche Medizinische Wochenschrift; 126, 1331-1334. http://dx.doi.org/10.1035/s-2001-18563

Lespade L, Bercion S. 2010. Theoretical study of the mechanism of inhibition of xanthine oxydase by flavonoids and gallic acid derivatives. Journal of Physical Chemistry, 114: 921-928. http://dx.doi.org/10.1021/jp9041809

Lioté F, Bardin T. 2007. Traitement de la goutte. Revue du Rhumatisme, $74: 160$ 167. http://dx.doi.org/10.1016./j/rhum/2006.1 2.005

Meli Lannang A, Lontsi D, Ngounou FN, Sondengam BL, Nkengfack AE, van Heerden FR, Assob JCN. 2006. Securidacaxanthone A, a heptaoxygenated xanthone from Securidaca longepedonculata. Fitoterapia; 77: 199-202. http://dx.doi.org/10.1016/j.fitote.2006.01 .006

Mikuls TR, Farrar JT, Bilker WB, Fernandes S, Schumacher Jr. HR, Saag KG. 2005. Gout epidemiology: results from the UK General Practice Research Database, 1990-1999. Ann Rheum Dis ; 64: 267-72. http://dx.doi.org/10.1136/ard.2004.0240 91

Ministère de la Santé Publique-Tchad. 2012. Annuaires des Statistiques Sanitaires. (Tome A, 26 ème édn). Ministère de la Santé Publique-Tchad : Tchad.
Muanda FN. 2010. Identification de polyphénols, évaluation de leur activité antioxydante et étude de leurs propriétés biologiques. Thèse de Doctorat, Paul verlaine-Metz, $294 \mathrm{p}$.

Ndiaye SY A, Fall AD, Ndiaye M, Sall O, SY GY, Bassène E, Dièye AM. 2016. Mise en évidence de l'activité antiinflammatoire des sous-fractions méthanoliques des feuilles de Moringa oleifera Lam. (Moringaceae) chez le rat. Int. J. Biol. Chem. Sci., 10(2) : 760-768. DOI : http://dx.doi.org/10.4314/ijbcs.v10i2.25

Nebie RHC, Yameogo RT, Belanger A, Sib FS. 2004. Salicylate de méthyle, constituant unique de l'huile essentielle de l'écorce des racines de Securidaca longepedunculata Fres du Burkina Faso. Comptes Rendus Chimie ; 7 : 1003-1006. DOI :

http://dx.doi.org/10.1016/j.crci.2003.12. 025

Okoli CO, Akah PA, Ezugworie U. 2006. Anti-inflammatory activity of extracts of root bark of Securidaca longipedunculata fres [polygalaceae]. Afr J.CAM; 2(3) : 54-63.

DOI: http://dx.doi.org/10.4314/ajtcam.v3i1.31 139

Philip CS, Thamara KD, Steven RB, Nigel CV. 2009. Bisdesmosidic Saponins from Securidaca longepedonculata Roots. Evaluation of Deterrency and Toxicity to Coleopteran Storage Pests. J. Agric. Food Chem., 57: 8860-8867. DOI: http://dx.doi.org/10.1021/jf901599j

Riegersperger M, Covic A, Goldsmith D. 2011. Allopurinol, uric acid, and oxidative stress in cardiorenal disease. International Urology and Nephrology; 43, 441-449. DOI: http://dx.doi.org/10.1007/s11255-0119929-6

Salawu O A, Chindo BA, Tijani AY, Adzu B. 2008. Analgesic, Anti-inflammatory, Anti-pyretic and Anti-plasmodial Effects of the Methanolic Extract of Crossopteryx febrifuga. J. Med. Plant 
Res., 2(8): $213-218 \quad$ DOI: http://dx.doi.org/10.5897/JMPR-

A26F9AA15267

Simon G, Dewelle J, Nacoulma O, Guissou $\mathrm{P}$, Kiss R, daloze $\mathrm{D}$, Braekman JC, 2003. Cytotoxic pentacyclic triterpènes from Combretum nigricans. Fitoterapia, 74: 339-344. DOI: http://dx.doi.org/10.1016/S0367326X(03)00046-7

Umamaheswari M, Asok Kumar, Somasundaram K. 2007. Xanthine oxidase inhibitory activity of some Indian medicinal plants. Journal of Ethnopharmacology 109: 547-551. DOI: http://dx.doi.org/10.1016/j.jep.2006.08.0 20

Wagner H, Bladt. 1996. Plant Drug Analysis, a Thin Layer Chromatography Atlas $\left(2^{\text {nd }}\right.$ edn). Springer-Verlag. Ed.: Berlin,
Yang XD, Li ZX, Yang SL, 2001. Xanthones from the stems of securidaca inappendiculata. Phytochemistry, 58: 1245-1249. DOI: http://dx.doi.org/10.1016/S00319422(01)00356-9

Zhan W, Doherty M, Pascual E, Bardin T, Barskova V, Conaghan P. 2010. EULAR evidence based recommendations for gout. Part II: management. Report of a task force of the EULAR Standing Committee for International Clinical Studies Including Therapeutics (ESCISIT). Ann Rheum Dis ; $\quad 65$ : 1312-1324. DOI : http://dx.doi.org/10.1136/ard.2006.0552 69 . 